\title{
Geografias da Diabetes Mellitus em Portugal: Como as Condições do Contexto Influenciam o Risco de Morrer
}

\author{
The Geography of Diabetes Mellitus in Portugal: How Context Influence the Risk \\ of Dying
}

\author{
Paula SANTANA ${ }^{1}$, Cláudia $\operatorname{COSTA}^{1}$, Adriana LOUREIRO ${ }^{1}$, João RAPOSO ${ }^{2}$, José Manuel BOAVIDA ${ }^{3}$ \\ Acta Med Port 2014 May-Jun;27(3):309-317
}

\section{RESUMO}

Introdução: A Diabetes Mellitus é um problema de saúde pública em crescimento em todo o mundo e também em Portugal. Pretendemos identificar a evolução do padrão geográfico desta causa de morte e a associação com a privação sociomaterial, em Portugal. Material e Métodos: Estudo ecológico transversal dos óbitos por Diabetes Mellitus ocorridos nos municípios portugueses em três períodos (1989-1993, 1999-2003 e 2006-2010). Aplicámos um modelo hierárquico bayesiano, de modo a obter a Razão Padronizada de Mortalidade Suavizada e o Risco Relativo, de mortalidade por diabetes associado à privação sociomaterial.

Resultados: Observámos os valores da Razão Padronizada de Mortalidade Suavizada mais elevados, em 1989-1993, nos municípios urbanos do litoral (80\% dos municípios com Razão Padronizada de Mortalidade Suavizada $\geq 161$, sendo $60 \%$ urbanos); em 20062010 verificámos o oposto, destacando-se, pelos valores de Razão Padronizada de Mortalidade Suavizada elevados, as áreas rurais loca-lizadas no interior sul do país (76,9\% dos municípios com Razão Padronizada de Mortalidade Suavizada $\geq 161$ sendo $69,2 \%$ rurais), principalmente no Alentejo. O Risco Relativo de mortalidade por Diabetes Mellitus aumenta com o aumento da vulnerabilidade associada às condições sociais e económicas da área de residência, principalmente nos dois últimos períodos (Risco Relativo: 1,00; IC95\%: 0,98-1,02).

Discussão: A Diabetes Mellitus apresenta um padrão geográfico marcado pela assimetria litoral-interior e urbano-rural, que tem vindo a alterar-se ao longo dos últimos vinte anos, verificando-se uma transição geográfica: $48 \%$ da população reside em municípios em que a Razão Padronizada de Mortalidade Suavizada aumentou nos últimos vinte anos, com destaque para as áreas rurais do interior de Portugal.

Conclusão: As Razões Padronizadas de Mortalidade Suavizada mais elevadas verificam-se, actualmente, em municípios rurais, com índice de privação sociomaterial mais elevado.

Palavras-chave: Demografia; Diabetes Mellitus/epidemiologia; Diabetes Mellitus/mortalidade; Factores Socioeconómicos; Portugal.

\section{ABSTRACT}

Introduction: Diabetes Mellitus is a public health problem that is on the increase throughout the world, including in Portugal. This paper aims to identify the changing geographic pattern of this cause of death in Portugal and its association with sociomaterial deprivation.

Material and Methods: This is a transversal ecological study of the deaths by Diabetes Mellitus in Portuguese municipalities in three periods (1989-1993, 1999-2003 and 2006-2010). It uses a Bayesian hierarchical model in order to obtain a smooth standardized mortality ratio and the relative risk of death by Diabetes Mellitus associated to sociomaterial deprivation.

Results: In 1989-1993, the highest smooth standardized mortality ratio values were found in coastal urban municipalities $(80 \%$ of municipalities with smooth standardized mortality ratio $\geq 161$, of which $60 \%$ are urban); in $2006-2010$, the opposite was found, with the highest smooth standardized mortality ratio values occurring in rural areas in southern inland regions $(76.9 \%$ of municipalities with smooth standardized mortality ratio $\geq 161$, of which $69.2 \%$ are rural), particularly the Alentejo. The relative risk of death by Diabetes Mellitus increases with vulnerability associated to social and economic conditions in the area of residence, and is significant in the last two periods (relative risk: 1.00; IC95\%: 0.98-1.02).

Discussion: Diabetes Mellitus presents a geographic pattern marked by coastal-inland and urban-rural asymmetry. However, this has been altering over the last twenty years. $48 \%$ of the population reside in municipalities where the smooth standardized mortality ratio has increased in the last twenty years, particularly in the rural areas of inland Portugal.

Conclusion: The highest smooth standardized mortality ratio are currently found in rural municipalities with the highest index of sociomaterial deprivation.

Keywords: Demography; Diabetes Mellitus/epidemiology; Diabetes Mellitus/mortality; Portugal; Socioeconomic Factors.

\section{INTRODUÇÃO}

A Diabetes Mellitus (DM) tipo 2 tem vindo a revelar-se uma patologia cada vez mais associada a grupos socioeconómicos mais desfavorecidos, sendo a sua prevalência e mortalidade mais elevadas em indivíduos em contextos de privação sociomaterial. ${ }^{1-13}$ Vários são os autores que têm investigado esta relação, apontando fatores de risco para a incidência da doença inerentes ao contexto individual, so- cial e geográfico do indivíduo. ${ }^{14-17}$ Lugares e comunidades envelhecidas, com baixa escolaridade, desemprego elevado, baixos rendimentos, más condições da habitação, fraca disponibilidade de infraestruturas que incentivem a adoção de estilos de vida saudáveis e com dificuldades de acesso aos cuidados de saúde transformam-se em ambientes vulneráveis, determinantes para a incidência da DM. ${ }^{18,19}$

\footnotetext{
1. Centro de Estudos em Geografia e Ordenamento do Território. Universidade de Coimbra. Coimbra. Portugal.

2. Associação Protectora dos Diabéticos de Portugal. Lisboa. Portugal.

3. Programa Nacional Para a Diabetes. Direção Geral de Saúde. Lisboa. Portugal.

Recebido: 26 de Abril de 2013 - Aceite: 22 de Setembro de 2013 | Copyright @ Ordem dos Médicos 2014
} 
A morbilidade e mortalidade da DM estão, deste modo, associados a ambientes obesogénicos, contextos que promovem comportamentos de risco, como dietas pobres e sobre-energéticas, inatividade física e, sedentarismo e consumo de tabaco. ${ }^{12,13,20-23}$ Pode concluir-se que os contextos de vivência do indivíduo podem influenciar os padrões de incidência, prevalência e mortalidade da DM, com consequências quer para o sistema de saúde, segurança social, quer para a qualidade de vida. ${ }^{14}$

A transição demográfica ${ }^{24}$ e epidemiológica estão associadas à transição nutricional, ${ }^{25}$ tendo consequências no aumento das doenças crónicas, onde se inclui a DM e as doenças cardiovasculares, ${ }^{26}$ sendo urgente adaptar as estruturas de saúde pública a esta realidade. ${ }^{27}$

No quadro Europeu, Portugal apresenta uma das taxas mais elevadas de mortalidade por DM: 97 óbitos por 100 000 habitantes dos 20 aos 79 anos. ${ }^{28}$ Nos últimos 15 anos registou-se um aumento do número total de óbitos, com o contributo principal de pessoas com 70 ou mais anos. ${ }^{29}$ No mesmo período, o número médio de anos de vida perdidos por DM abaixo dos 70 anos apresenta um ligeiro aumento (8,4 anos em 2010), sendo superior para os homens. ${ }^{29} \mathrm{Ou}$ seja, ocorreram mais mortes e mais cedo (em idades mais jovens).

Portugal é o segundo país da Europa com maior prevalência de DM: 12,7 indivíduos com DM por cada 100 habitantes em 2011,28 estimando-se para 2030 um aumento para $15,2 \%$. Segundo o Observatório Nacional da Diabete $^{30}$ a prevalência de DM em 2010 é maior na população idosa (27,1\% dos indivíduos dos 60 aos 79 anos), nos indivíduos com nível educacional mais baixo (representando os analfabetos $30,3 \%$ dos indivíduos com DM) e nos obesos $(39,6 \%$ do total). O padrão é semelhante no Relatório de $2012 .{ }^{31}$

Em Portugal, não existe até hoje informação desagregada por área de residência que permita estabelecer associação entre áreas de residência (rural, urbana) e ambientes obesogénicos. Por outro lado, ainda não foi avaliada a influência das condições sociais e económicas da área de residência na taxa de mortalidade da DM. O objectivo deste trabalho é avaliar a evolução dos padrões geográficos de mortalidade e do risco relativo de morrer por DM em Portugal, nos últimos vinte anos.

\section{MATERIAL E MÉTODOS}

Aplicámos um estudo ecológico transversal, correspondendo a unidade de análise aos municípios de Portugal Continental. Analisámos os óbitos por DM (CID9: 250, CID10: E10-E14), que ocorreram nos períodos de 19891993, 1999-2003 e 2006-2010, estratificados por género e grupo etário (< 5 anos, 5-14 anos, 15-49 anos, 50-69 anos, $70-74$ anos e $\geq 75$ anos), provenientes dos registos vitais e recolhidos pelo Instituto Nacional de Estatística (INE). Devido a problemas de codificação do local de residência, sexo e grupo etário, a proporção de óbitos não utilizados neste estudo variou entre 0,1\% (2006-2010) e 2,5\% (1989-1993).

A população em estudo é relativa aos indivíduos resi- dentes nos municípios de Portugal Continental, estruturados por género e pelos mesmos grupos etários dos óbitos, registados nos Censos de 1991 e 2001 e estimados pelo INE para 2008.

Construímos um Índice de Privação Sociomaterial para os anos 1991, 2001 e 2011 (com informação dos Censos, INE), adaptado a partir da metodologia de Carstairs et al. ${ }^{32}$ É um indicador composto, agregador de três dimensões; duas relativas a condições socioeconómicas (analfabetismo e desemprego) e uma relativa às condições do alojamento (alojamentos sem retrete). Este índice traduz-se em valores positivos, representativos de áreas com privação alta, e negativos, representativos de áreas com privação baixa.

Construímos, ainda, um indicador relativo à Tipologia de Áreas de Residência à escala do Município, adaptando o que foi produzida pelo INE, em 2009 para a escala da Freguesia; com o objetivo de avaliar a hipótese da existência (ou não) de um padrão urbano/rural de mortalidade por DM.

O indicador de mortalidade que utilizámos neste estudo foi a Razão Padronizada de Mortalidade (RPM), com base no método indirecto, tomando como referência a estrutura populacional de Portugal Continental, por género e grupo etário. A RPM é dependente do tamanho da população e as áreas com pouca população tendem a ter valores estimados muito elevados. Para controlar a variabilidade na estimação do risco de mortalidade, optámos por aplicar o modelo hierárquico bayesiano proposto por Besag et $\mathrm{al}^{33}$ e calculámos a Razão Padronizada de Mortalizada suavizada (RPMs). Este modelo tem em conta dois tipos de efeitos aleatórios - espacial e heterogéneo. O primeiro tem em conta a auto-correlação espacial dos dados, enquanto o segundo se refere à variabilidade não-espacial dos dados (as estimações obtidas entre áreas são independentes entre si). ${ }^{34}$ Com base na RPMs, foi ainda calculada a significância estatística do risco de morrer por DM, definida por uma RPMs superior a 100.

De modo a analisar a associação entre a mortalidade por DM e a privação sociomaterial, foi aplicado um modelo de regressão ecológico, que introduz a privação sociomaterial como co-variável contínua. ${ }^{34}$

Tabela 1 - População Residente em Portugal Continental

\begin{tabular}{|c|c|c|c|c|}
\hline & & 1991 & 2001 & 2008 \\
\hline & e áreas & 275 & 278 & 278 \\
\hline & Mínimo & 2052 & 1924 & 1697 \\
\hline$\stackrel{\text { Tू }}{=}$ & Máximo & 663394 & 564657 & 489562 \\
\hline$\underset{0}{T}$ & Média & 34094 & 35501 & 36458 \\
\hline & Mediana & 16207 & 16356 & 16312 \\
\hline & e Média & 37 & 39 & - \\
\hline
\end{tabular}


Para a operacionalização de ambos os modelos, utilizou-se o método INLA (Integrated Nested Laplace Approximations), através da biblioteca INLA, no software R.2.15.2.

\section{RESULTADOS}

A população residente de Portugal Continental distribui-se heterogeneamente por 278 municípios (Tabela 1). No primeiro período em análise apenas foram tidos em conta 275 municípios. Só em 1998 foram constituídos três novos municípios. Em 2008, a população por município variou entre 1697 e 489562 habitantes, sendo a mediana de 16312 habitantes, valor bastante distante da média (36 458), confirmando a variabilidade demográfica do país.

Os óbitos por DM têm vindo a aumentar e representam atualmente (2006-2010) 4,1\% dos óbitos totais (em 19891993 representavam 2,8\%), sendo os valores mais elevados nas mulheres, quando comparados com os homens; em 1989-1993 a diferença era de 18\%, e em 2008-2010 de $12 \%$ (Tabela 2).

A taxa bruta de mortalidade por DM aumentou, entre 1989-1993 e 2006-2010, de 1,40 óbitos para 2,0 por 1000 habitantes.

A privação sociomaterial evidencia nos três períodos um padrão estável: um litoral com valores mais baixos de privação sociomaterial, persistindo valores elevados nos municípios rurais do interior do País. No entanto, verifica-se uma evolução demográfica ao longo dos anos: diminuiu a população a residir nos quintis extremos (mais alto e mais baixo) de privação sociomaterial e aumentou nos quintis intermédios de privação (Tabela 3).

A RPMs por DM apresenta, genericamente, um padrão geográfico marcado pela assimetria litoral-interior ao qual se sobrepõe o padrão norte-sul (Fig. 1). Todavia, ao longo dos vinte anos verifica-se que ocorreu uma alteração substantiva neste padrão. Em 1989-1993 os valores da RPMs são elevados e muito elevados nos municípios do litoral, especialmente concentrados nas Áreas Metropolitanas de Lisboa e Porto, e baixos e muito baixos no interior, especialmente no Centro, Norte e Algarve. No período 1999-2003 o padrão altera-se, verificando-se valores mais baixos que os reportados anteriormente nas Áreas Metropolitanas e a concentração de valores elevados no sul do País (Alentejo, Oeste, Médio Tejo e Lezíria). Em 2006-2010, este padrão acentua-se, confirmando-se os valores de RPMs baixos e muito baixos na Área Metropolitana de Lisboa, em contraste com os valores altos e muito altos nos municípios do interior sul do Alentejo, invertendo o padrão observado vinte anos antes. O Algarve mantém os valores baixos de RPMs ao longo dos vinte anos da análise.

Considerando a evolução da RPMs entre 1989-1993 e 2006-2010, registou-se um agravamento em 1/3 dos municípios, com 7\% a duplicarem os valores de RPMs (Fig. 2). Estes agravamentos concentram-se, genericamente, no interior do país. Em sentido contrário, observa-se diminuição, para quase metade do valor da RPMs, nomeadamente nas Áreas Metropolitanas de Lisboa e Porto e outras áreas urbanas do litoral. Todavia, apenas $19 \%$ da população reside em municípios cuja RPMs melhorou, e que passaram a ser inferiores ao padrão do Continente. De facto, apesar dos ganhos observados em algumas áreas urbanas, verifica-se que a maioria da população (48\%) reside em municípios cuja RPMs aumentou.

A mortalidade por DM tem padrões distintos entre Homens e Mulheres (Fig.s 3 e 4). Em 1989-1993 o padrão li-

Tabela 2 - Óbitos por período e género

\begin{tabular}{|c|c|c|c|}
\hline & 1989 - 1993 & 1999 - 2003 & $2006-2010$ \\
\hline Total & 13502 & 18142 & 20229 \\
\hline Homens & 5484 & 7639 & 8873 \\
\hline Mulheres & 8018 & 10503 & 11356 \\
\hline Ignorado* & 334 & 116 & 29 \\
\hline
\end{tabular}

* Somatório de casos em que não foi possível identificar o sexo, grupo etário ou concelho de residência. Fonte: INE - Estatísticas da Saúde (dados não publicados).

Tabela 3 - População Residente nos municípios, por quintis de privação sociomaterial

\begin{tabular}{|c|c|c|c|c|}
\hline \multirow{2}{*}{$\begin{array}{l}\text { Privação Sociomaterial } \\
\text { (em quintis) }\end{array}$} & \multicolumn{3}{|c|}{ População Residente (\%) } & \multirow{2}{*}{$\begin{array}{l}\text { Variação entre } \\
1991 \text { e } 2011 \text { (\%) }\end{array}$} \\
\hline & 1991 & 2001 & 2011 & \\
\hline $1<$ privação & 60,9 & 62,7 & 42,8 & $-29,7$ \\
\hline 2 & 15,1 & 20,0 & 29,8 & 97,8 \\
\hline 3 & 11,4 & 5,8 & 17,8 & 56,4 \\
\hline 4 & 6,7 & 5,2 & 5,2 & $-22,3$ \\
\hline 5 > privação & 6,1 & 6,3 & 4,5 & $-26,0$ \\
\hline
\end{tabular}


$1989-1993$

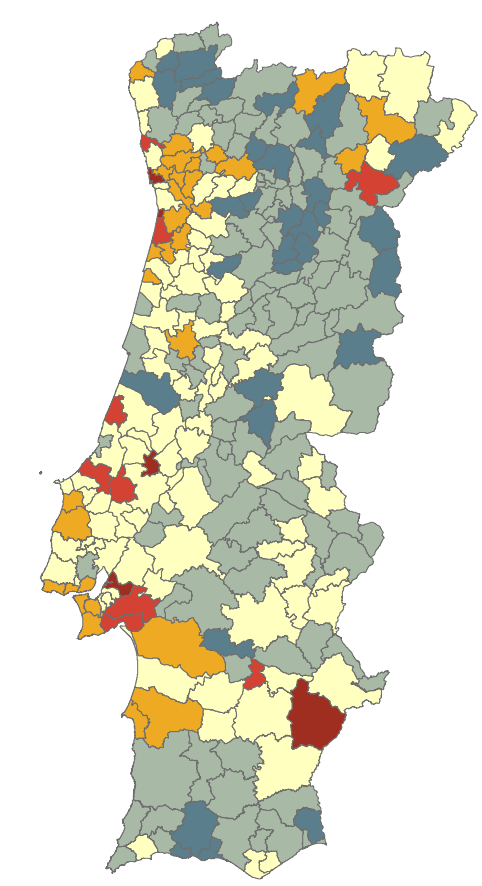

$1999-2003$

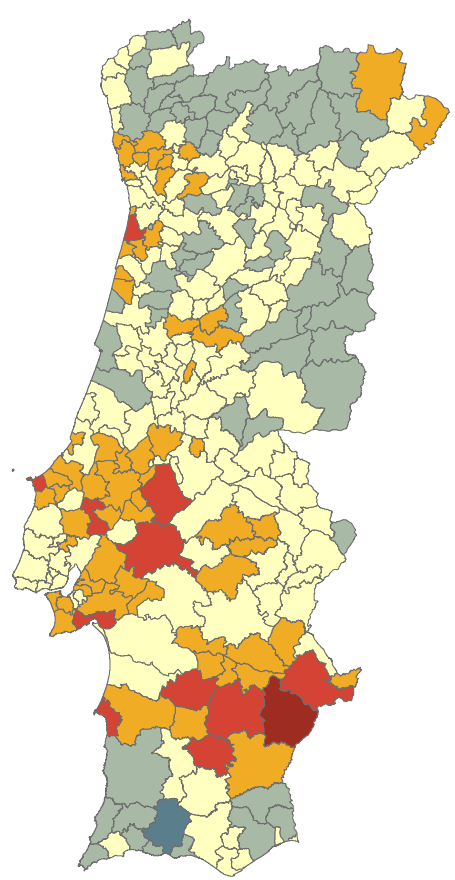

$2006-2010$

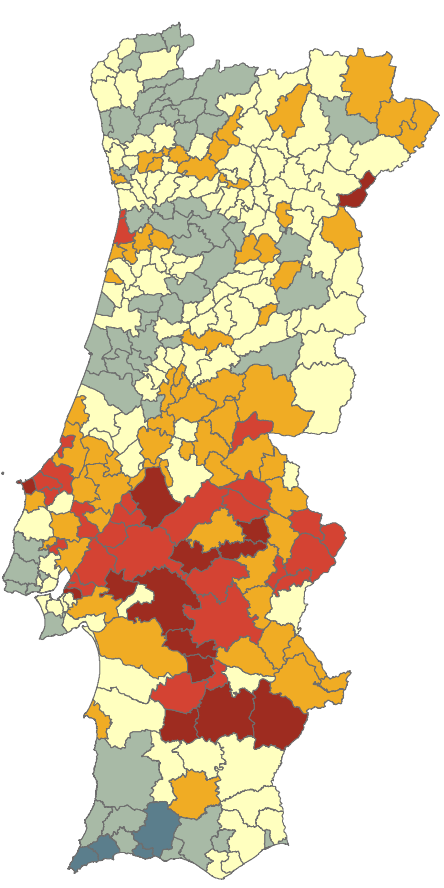

\section{Legenda:}

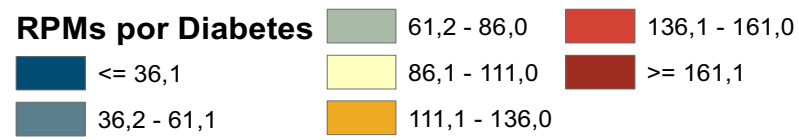

Figura 1 - RPMs por Diabetes Mellitus em Portugal Continental - Total. Fonte: Elaboração própria com base em INE - Estatísticas da Saúde (dados não publicados)

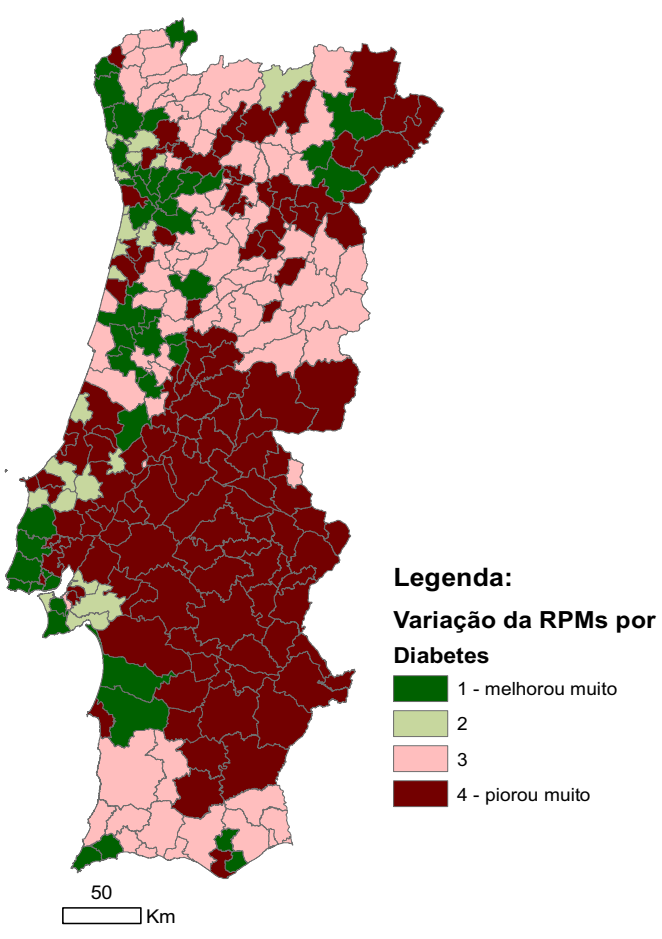

Figura 2 - Evolução da RPMs por Diabetes Mellitus em Portugal Continental (1989-1993 e 2006-2010).

Fonte: Elaboração própria com base em INE - Estatísticas da Saúde (dados não publicados) toral, de RPMs com valores elevados, principalmente junto das áreas metropolitanas, era mais acentuado nas mulheres, quando comparado com o dos homens. Nos períodos seguintes o padrão de mortalidade revela maior concentração nos homens, quando comparado com o das mulheres. Por outro lado, os valores de RPMs mais elevados são observados nas mulheres, embora essa discrepância se tenha vindo a atenuar ao longo dos vinte anos em análise (1989-1993: Homens = 151,9 e Mulheres = 218,9; 20062010: Homens = 196,2; Mulheres = 196,8).

Como foi referido anteriormente, as áreas de risco de morte por DM têm aumentado no período em análise, principalmente em municípios rurais, incluindo municípios próximos das áreas metropolitanas, e no centro-litoral e interior do Alentejo (Fig. 5). Acresce referir que no último período do estudo quase metade dos municípios do Continente são considerados como áreas de risco muito elevado. No entanto, devido à transição epidemiológica verificada nos municípios mais urbanizados (e principalmente da Área Metropolitana de Lisboa), a população a residir em áreas de risco diminuiu $(1991=44,7 \% ; 2011=37,4 \%)($ Tabela 4$)$.

Também na análise temporal do risco de morrer se verifica a alteração do padrão geográfico identificado anteriormente: entre 1989-1993 e 2006-2010 a probabilidade de morrer diminuiu nas áreas urbanas, incluindo quase todos os municípios da área metropolitanas de Lisboa e alguns da do Porto, e aumenta nas áreas rurais (Fig. 5). 
$1989-1993$

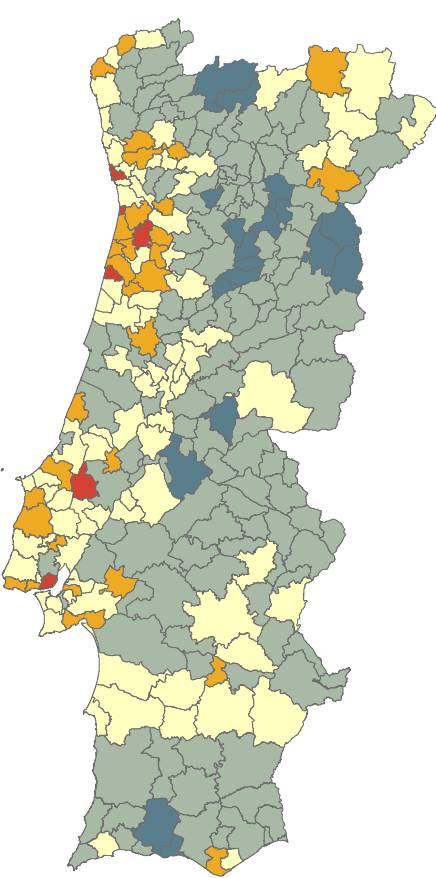

$1999-2003$

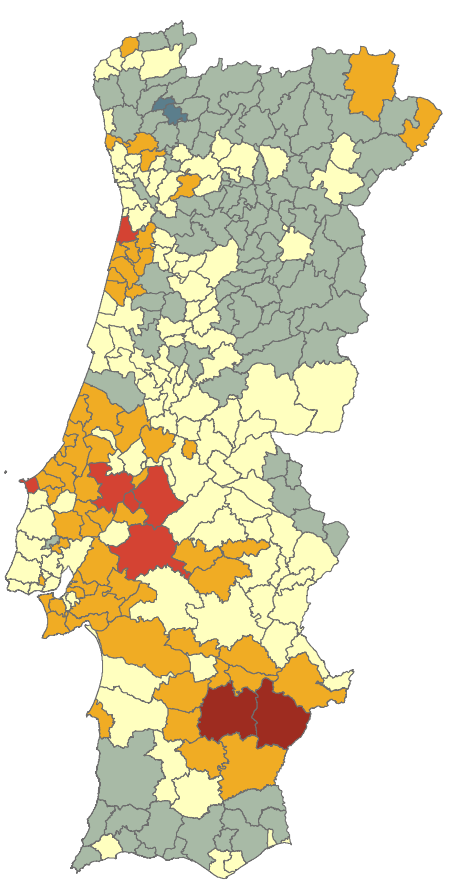

\section{Legenda:}

\begin{tabular}{l|l|l|}
$\begin{array}{l}\text { RPMs por Diabetes } \\
\text { Homens }\end{array}$ & $38,6-62,5$ & $110,6-134,6$ \\
\hline 51,5-38,5 & $62,6-86,5$ & $134,7-158,6$ \\
\hline & $86,6-110,5$ & $>=158,7$
\end{tabular}

Figura 3 - RPMs por Diabetes Mellitus em Portugal Continental - Homens.

Fonte: Elaboração própria com base em INE - Estatísticas da Saúde (dados não publicados).

1989 - 1993

1999 - 2003

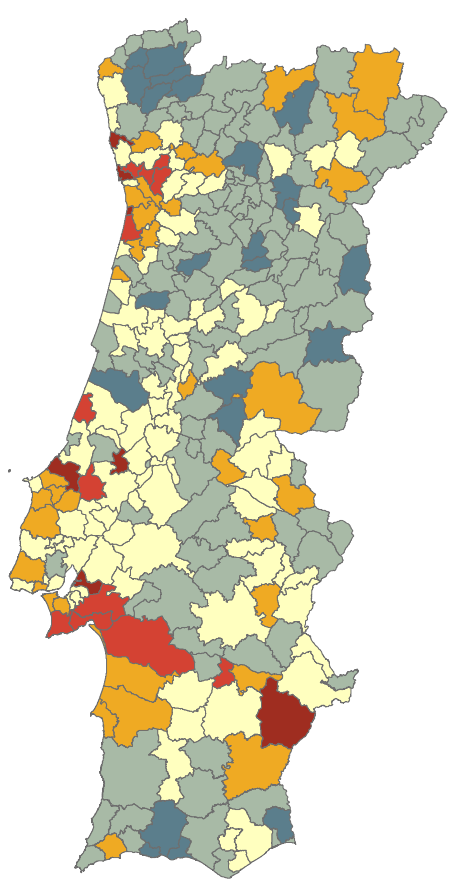

Legenda:

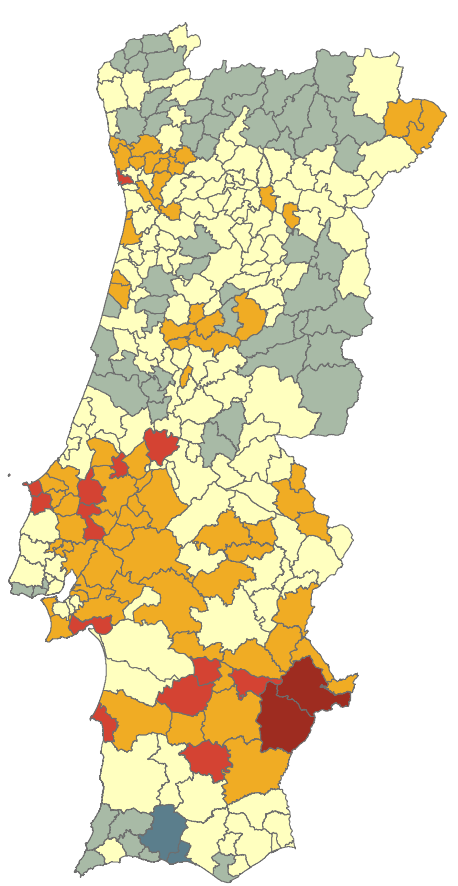

$110,6-134,6$

$134,7-158,6$

$>=158,7$

\begin{tabular}{l|l|l|}
$\begin{array}{l}\text { RPMs por Diabetes } \\
\text { Mulher }\end{array}$ & $38,6-62,5$ & \\
Mulher & & $110,6-134,6$ \\
\hline$<=38,5$ & $62,6-85,6$ & $134,7-158,6$ \\
\hline & $85,7-110,5$ & $>=158,7$
\end{tabular}

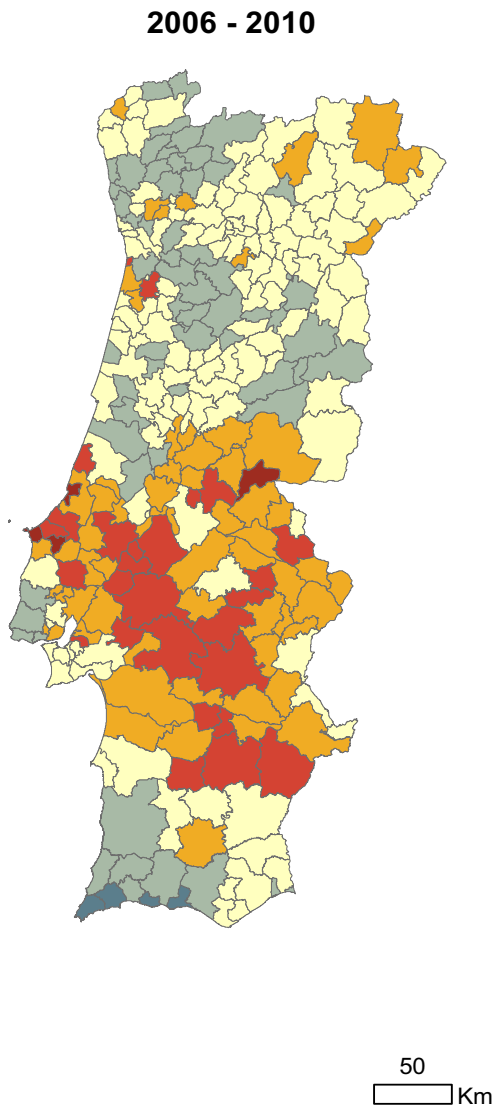

$2006-2010$

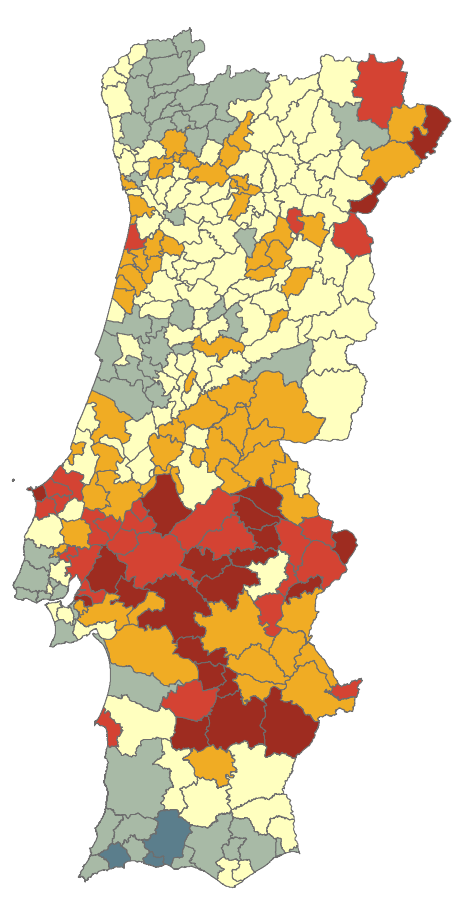

50

Figura 4 - RPMs por Diabetes Mellitus em Portugal Continental - Mulheres.

Fonte: Elaboração própria com base em INE - Estatísticas da Saúde (dados não publicados). 

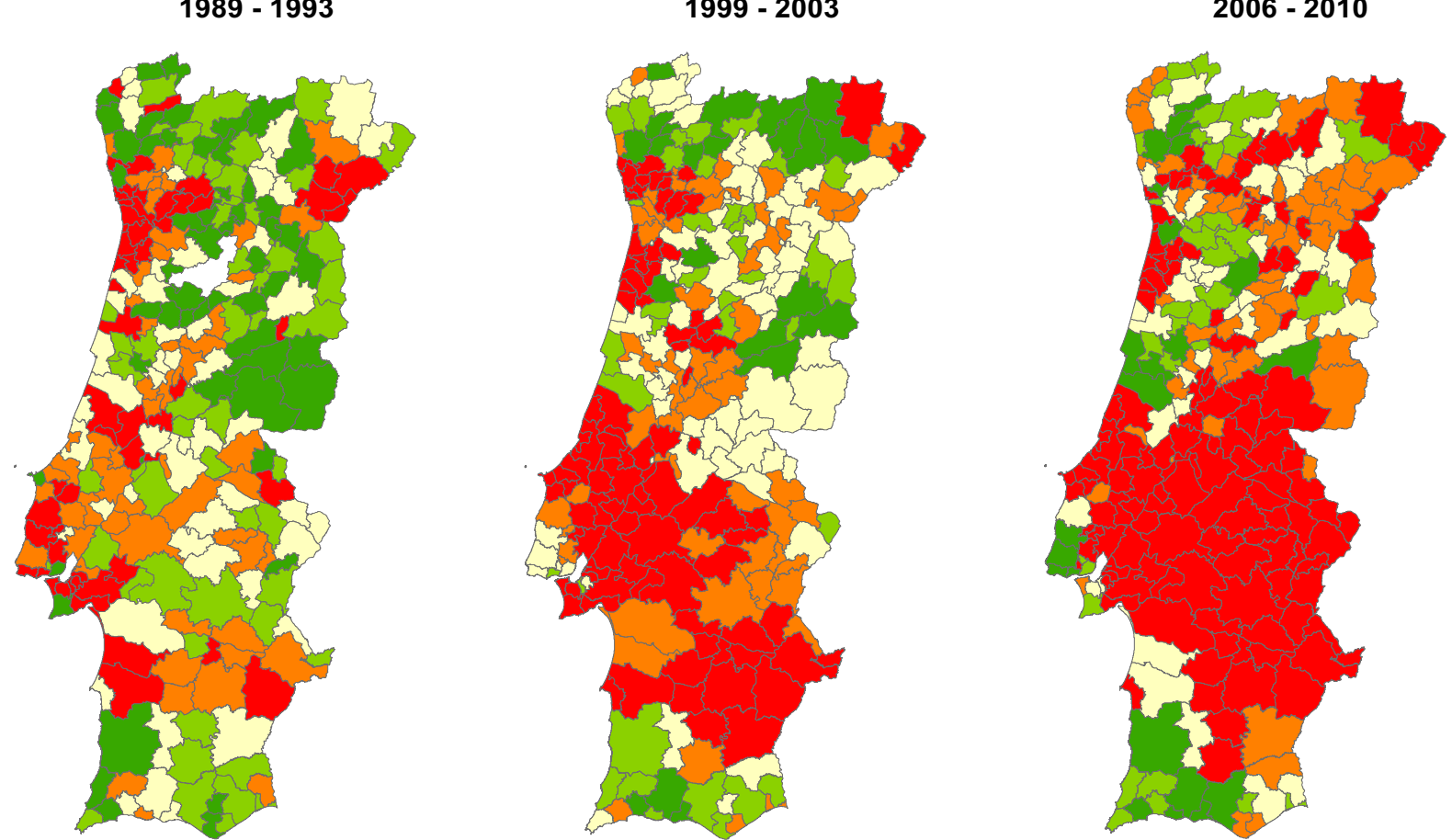

\section{Risco de Morrer}

\begin{tabular}{c|l|l|l|} 
por Diabetes & $0,008-0,070$ \\
$0,000-0,007$ & $\square, 279-0,675$ \\
\hline & $0,071-0,278$ & $0,676-1,000$
\end{tabular}

Figura 5 - Risco de Morrer por Diabetes Mellitus - Total.

Fonte: Elaboração própria com base em INE - Estatísticas da Saúde (dados não publicados)

Tabela 4 - População a residir em áreas de risco de morte por Diabetes Mellitus elevado

\begin{tabular}{ccccc}
\hline & & 1991 & 2001 & 2011 \\
\hline População & $N^{\circ}$ & 4193346 & 3233075 & 3787458 \\
Residente & $\%$ & 44,7 & 32,8 & 37,4 \\
\hline
\end{tabular}

Fonte: Elaboração própria com base em INE - Estatísticas da Saúde (dados não publicados) e Censos (1991, 2001 e 2011).

Tabela 5 - Risco Relativo da Associação entre Privação Sociomaterial e RPMs por Diabetes Mellitus

\begin{tabular}{|c|c|c|c|c|c|c|}
\hline \multirow{2}{*}{$\begin{array}{c}\text { Privação } \\
\text { Sociomaterial }\end{array}$} & \multicolumn{2}{|c|}{1989 - 1993} & \multicolumn{2}{|c|}{$1999-2003$} & \multicolumn{2}{|c|}{$2006-2010$} \\
\hline & $\mathbf{R R}$ & IC95\% & $\mathbf{R R}$ & IC95\% & $\mathbf{R R}$ & IC95\% \\
\hline Total & 0,94 & $0,92-0,96$ & 1,00 & $0,98-1,02$ & 1,00 & $0,98-1,02$ \\
\hline Homens & 0,92 & $0,90-0,94$ & 1,00 & $0,97-1,02$ & 1,00 & $0,98-1,03$ \\
\hline Mulheres & 0,95 & $0,93-0,98$ & 1,00 & $0,99-1,03$ & 1,00 & $0,98-1,02$ \\
\hline
\end{tabular}

Fonte: Elaboração própria com base em INE - Estatísticas da Saúde (dados não publicados) e Censos (1991, 2001 e 2011).

Tabela 6 - População e Mortalidade por Diabetes Mellitus por níveis de privação sociomaterial

\begin{tabular}{|c|c|c|c|c|c|}
\hline Privação & Mortalidade & 1989 - 1993 & $1999-2003$ & $2006-2010$ & $\begin{array}{c}\text { Variação entre } \\
1989 \text { - } 1993 \text { e } 2006 \text { - } 2010\end{array}$ \\
\hline mais & mais & $\begin{array}{l}390846 \\
4,2 \%\end{array}$ & $\begin{array}{l}453963 \\
4,6 \%\end{array}$ & $\begin{array}{l}725073 \\
7,2 \%\end{array}$ & $71,6 \%$ \\
\hline menos & menos & $\begin{array}{c}3286417 \\
35,1 \%\end{array}$ & $\begin{array}{c}5113337 \\
51,8 \%\end{array}$ & $\begin{array}{c}4871195 \\
48,1 \%\end{array}$ & $37,1 \%$ \\
\hline
\end{tabular}

Fonte: Elaboração própria com base em INE - Estatísticas da Saúde (dados não publicados) e Censos (1991, 2001 e 2011). 
De facto, parece existir uma associação positiva e entre a privação sociomaterial e a RPMs, embora apenas nos dois últimos períodos do estudo, tanto para o total da população, como para ambos os géneros (Tabela 5). Apesar de a associação não ser significativa no primeiro período, é interessante verificar que, em termos de escala temporal, o risco relativo aumenta quando se observa a relação entre a DM e a privação.

Observando o comportamento dos municípios de maior privação e maior mortalidade, nos três períodos, verifica-se que a população quase que duplicou, representando em 2006-2010 cerca de 7,2\% da população portuguesa. Em sentido oposto, $48,1 \%$ da população reside em municípios de baixa privação e baixa RPMs por DM (Tabela 6).

Em síntese: a mortalidade (RPMs) por DM tem vindo a intensificar-se ao longo dos últimos vinte anos, mas com maior expressão nos municípios rurais do interior. Também a probabilidade de morrer por DM, em 2006-2010, é elevada e muito elevada nas regiões do Alentejo, Beira Interior Sul, Oeste, Médio Tejo e Lezíria, estando principalmente associada a áreas de maior envelhecimento populacional e de maior ruralidade. Ao longo dos últimos vinte anos verificou-se uma associação entre a RPMs e o índice de privação sociomaterial da área de residência, apesar dessa associação ser apenas significativa nos dois últimos períodos da análise. Ou seja, a mortalidade por DM aumenta com o aumento da privação de condições sociais e económicas básicas, expressas na baixa escolaridade, trabalho não qualificado e má qualidade da habitação.

\section{DISCUSSÃO}

À semelhança do que outros autores tornaram evidente para a DM (particularmente a de tipo 2), também neste estudo parece poder concluir-se que, em 2006-2010, esta patologia está associada a áreas rurais e grupos socioeconómicos mais desfavorecidos, sendo o risco de morte mais elevado em indivíduos e contextos de privação sociomaterial. Todavia, vinte anos antes (1989-1993) o padrão era marcadamente urbano e não tinha sido encontrada associação entre a RPMs por DM e a privação sociomaterial. Em vinte anos, a RPMs por DM aproximou-se do padrão observado em alguns países europeus. Ou seja, verificou-se, também em Portugal, que as condições de vida individual (às condições precárias das habitações, aos baixos níveis de instrução e de rendimento) e coletiva (ruralidade) associada à DM constituem-se como determinantes sociais da DM, amplificando o contributo dos ambientes obesogénicos.

À semelhança do que tinha acontecido noutros países da Europa, as políticas públicas relativas à habitação, emprego e de oferta e acesso a bens e serviços tiveram impactos na redução da mortalidade infantil e no aumento da esperança de vida à nascença. ${ }^{24}$ Por outro lado, não deve ser escamoteado o efeito da transição nutricional, ${ }^{25}$ resultado da urbanização/globalização e do crescente consumo de alimentos industrializados e pré-preparados na alteração da dieta alimentar, e o aparecimento do binómio excesso de peso/obesidade. Todos estes fenómenos, com particular destaque para o envelhecimento da população, têm consequências no aumento da DM e outras doenças crónicas associadas, como as cardiovasculares. ${ }^{26}$

De fato, a DM é um significativo problema de saúde pública em Portugal, principalmente pela associação com os problemas de mortalidade e morbilidade cardiovascular, podendo vir a ganhar dimensão epidémica em 2030. A prevenção primária poderá vir a contrariar esta tendência. ${ }^{27}$ Todavia, importa considerar alguns aspetos que podem ter contribuído para o aumento da prevalência da DM em Portugal e que têm aumentado nos últimos vinte anos: em primeiro lugar, o envelhecimento da população (o número de pessoas com 65 ou mais anos de idade passou de 1 693493 - 16,4\% - para 2011064 - 19\%, entre 1991 e em 2011); em segundo lugar o excesso de peso e a obesidade (entre 1996 e 2006 variação positiva de 55,5\% para os Homens e 45,7\% para as Mulheres; em 2006, 16,5\% de população residente era obesa e 53,4\% apresentava excesso de peso). ${ }^{35}$ Por fim, o impacte das desigualdades geográficas e das condições sociais e económicas da área de residência, quer na mortalidade quer na prevalência, impacte esse que importa avaliar.

Sabe-se que ambientes obesogénicos têm contribuído para o aumento do excesso de peso e obesidade. Verificou-se um aumento de $46 \%$ na ingestão de calorias, entre 1961 e 2009 , sendo o consumo actual de $3617 \mathrm{kcal} / \mathrm{per}$ capita/ dia, valor considerado elevado e acima da média europeia. ${ }^{36}$ Simultaneamente observam-se valores percentuais de sedentarismo elevados - em $2009,55 \%$ da população portuguesa afirmou não praticar qualquer atividade física (incluindo trabalho), sendo superiores à média europeia. ${ }^{37}$ Em Portugal, não existe até hoje informação desagregada por área de residência que permita estabelecer associação entre áreas mais pobres e ambientes obesogénicos. Por outro lado, este é o primeiro estudo que permite avaliar a influência das condições geográficas e socioeconómicas da área de residência na taxa de mortalidade da DM em Portugal.

Tem havido trabalhos de investigação científica noutros países que demonstram que a prevenção ou, pelo menos, o retardamento da DM de tipo 2 é possível. Em indivíduos geneticamente vulneráveis ou com alto risco, a combinação de perda de peso moderada, aumento da atividade física e recomendações alimentares/dietéticas pode levar a uma redução de $60 \%$ na incidência de DM. Ou seja, intervenções que têm uma forte componente de educação para comportamentos e estilos de vida saudáveis podem atenuar o impacto das desigualdades sociais no desenvolvimento da DM. Todavia, a educação pessoal e a mudança de comportamentos apenas serão suscetíveis de ser estimuladas em ambientes que criem oportunidade para comportamentos saudáveis (disponibilidade de lojas de produtos alimentares frescos - como vegetais e frutas -, na área de residência; áreas caminháveis seguras, e.g.).

Em 2003, segundo o Eurobarómetro, ${ }^{38}$ apenas metade dos portugueses refere que o local onde moram oferece 
oportunidades para a prática de atividade física e possui associações e clubes desportivos. Em 2009 aumentou a percentagem de indivíduos que responde positivamente. Os autarcas (Políticas Municipais) são, por isso, parceiros preferenciais se o objetivo é alterar a relação entre pobreza/diabetes/pobreza, já que a DM é consequência mas também causa de pobreza.

De fato, os fatores sociais e económicos têm uma carga pesada na vulnerabilidade dos indivíduos para o desenvolvimento da DM e, ainda, das suas complicações. $\mathrm{O}$ desafio que se coloca, para além da mudança de atitude e comportamentos, é o de criar programas públicos voltados para a prevenção da DM, das suas complicações e consequências, desenvolvendo e avaliando formas de abordar os fatores subjacentes que tornam os indivíduos vulneráveis. ${ }^{27}$ Por exemplo, encontrando programas/ações/medidas para reduzir os ambientes obesogénicos. A base da Estratégia da OMS sobre Dieta, Atividade Física e Saúde já está vertida no Programa Nacional para a Diabetes, sendo necessário usar a política para mudar os ambientes obesogénicos.

\section{CONCLUSÕES}

Ao longo dos últimos vinte anos o número de registos de óbitos e o número médio de anos de vida perdidos por DM têm vindo a aumentar, podendo concluir-se que em 2006-2010 ocorreram mais mortes e que estas ocorreram mais cedo, quando comparadas com as de 1989-1993. O padrão geográfico alterou-se: A RPMs diminuiu nas áreas urbanas do litoral (principalmente nas Áreas Metropolitanas de Lisboa e Porto) e aumentou nas áreas rurais, principalmente a sul do Rio Tejo; aumentou a probabilidade de morrer por DM nas áreas rurais. Nos últimos anos verificou-se que o risco relativo de mortalidade por DM aumenta com a vulnerabilidade associada às condições sociais e económicas da área de residência.

\section{CONFLITOS DE INTERESSE}

Nada a declarar.

\section{FONTES DE FINANCAMENTO}

Artigo realizado com o apoio do projecto Geografia do Estado de Saúde - Uma aplicação do Índice de Saúde da População nos últimos 20 anos (GeoHealthS), financiado pela Fundação para a Ciência e Tecnologia (PTDC/CS-GEO/122566/2010) e do Observatório Nacional da Diabetes.

13. Connolly V, Unwin N, Sherriff P, Bilous R, Kelly W. Diabetes prevalence and socioeconomic status: a population based study showing increased prevalence of type $2 \mathrm{DM}$ in deprived areas. J Epidemiol Community Health. 2000;54:173-7.

14. Whiting D, Unwin N, Roglic G. Diabetes: equity and social determinants In: Blas E, Kurup A, editors. Equity, social determinants and public health programs. World Health Organization; 2010. p.77-94.

15. Maddigan S, Feeny D, Majumdar S, Farris K, Johnson J. Understanding the determinants of health for people with type 2 diabetes. Am J Public Health. 2006;96:1649-55

16. Schulz A, Zenk S, Odoms-Young A, Hollis-Neely T, Nwankwo R, Locket $M$, et al. Healthy eating and exercising to reduce diabetes: exploring the potential of social determinants of health frameworks within the context of community-based participatory diabetes prevention. Am J Public Health. 2005;95:645-51.

17. Brown A, Ettner S, Piette J, Weinberger M, Gregg E, Shapiro M, et al. Socioeconomic position and health among persons with DM: a conceptual framework and review of the literature. Epidemiol Rev. 2004;26:63-77.

18. Schmitz N, Nitka D, Gariepy G, Malla A, Wang J, Boyer R, et al Association between neighborhood-level deprivation and disability in a community sample of people with diabetes. Diabetes Care. 2009;32:1998-2004

19. Weng C, Coppinit D, Sönksen P. Geographic and social factors are related to increased morbidity and mortality rates in diabetic patients. Diabet Med. 2000;17:612-7.

20. Cooper A, Sebire S, Montgomery A, Peters T, Sharp D, Jackson N, et al. Sedentary time, breaks in sedentary time and metabolic variables in people with newly diagnosed type 2 diabetes. Diabetologia. 2012;55:589-99.

21. Santana $P$, Santos $R$, Nogueira $H$. The link between local environment and obesity: a multilevel analysis in the Lisbon Metropolitan Area, Portugal. Soc Sci Med. 2009;68:601-9.

22. Yoon K, Lee J, Kim J, Cho J, Choi Y, Ko S, et al. Epidemic obesity and type 2 diabetes in Asia. Lancet. 2006;368:1681-8.

23. Stern M, Gonzalez C, Mitchell BD, Villalpando E, Haffner S, Hazuda H Genetic and environmental determinants of type II diabetes in Mexico City and San Antonio. Diabetes. 1992;41:484-92.

24. Friederiksen $\mathrm{H}$. Feedbacks in economic and demographic transition. 
Science. 1969;166:837-47.

25. Popkin BM. Nutritional patterns and transitions. Populations and Development Rev. 1993;19:138-57.

26. Omran AR. The epidemiologic transition. A theory of the epidemiology of population change. Milbank Mem Fund Q. 1971;49:509-38.

27. Santana P. Geografias da saúde e do desenvolvimento - evolução e tendências em Portugal. Coimbra: Edições Almedina; 2005.

28. International Diabetes Federation; c2012; [consultado 2012 Nov 11]. Disponível em: http://www.idf.org/diabetesatlas/5e/Update2012.

29. Comissão Europeia - Eurostat; c2012; [consultado 2012 Nov 11] Disponível em: http://epp.eurostat.ec.europa.eu/portal/page/portal/ statistics/search_database.

30. Boavida JM, Fragoso de Almeida JP, Massano Cardoso S, Sequeira Duarte J, Duarte R, Ferreira H, et al. Diabetes: factos e números 2011 - Relatório anual do Observatório Nacional da Diabetes. Lisboa: OND, Sociedade Portuguesa de Diabetologia; 2012.

31. Correia L, Boavida JM, Fragoso de Almeida JP, Massano Cardoso S, Dores J, Sequeira Duarte J, et al. Diabetes: factos e números 2012 Relatório anual do Observatório Nacional da Diabetes. Lisboa: OND,
Sociedade Portuguesa de Diabetologia; 2013.

32. Carstairs V, Morris R. Deprivation and health in Scotland. Aberdeen: Aberdeen University Press; 1991.

33. Besag J, York J, Mollié A. Bayesian image restoration, with two applications in spatial statistics. Ann Inst Stat Math. 1991;43:1-59.

34. Santana P, Costa C, Mari-dell'OImo M, Palència L, Gotsens M, Borrell C. Socioeconomic inequalities and mortality: exploring possible relationships in the Lisbon Metropolitan Area. Eur $\mathrm{J}$ Epidemiol. 2012;27:62.

35. Inquérito Nacional de Saúde 2005-2006. Lisboa: Instituto Nacional de Saúde Doutor Ricardo Jorge e Instituto Nacional de Estatística; 2006.

36. Food and Agriculture Organization of the United Nations; c2012; [consultado 2012 Nov 11]. Disponível em: http://faostat3.fao.org/home/ index.html\#VISUALIZE.

37. Comissão Europeia. Special Eurobarometer 334 / Wave 72.3: Sport and Physical Activity. Bruxelas: TNS Opinion \& Social; Comissão Europeia.; 2010.

38. Comissão Europeia. Special Eurobarometer 183-6/ Wave 58.2: Physical Activity. Bruxelas: European Opinion Research Group EEIG; 2003. 


\section{Geografias da Diabetes Mellitus em Portugal: Como as Condições do Contexto Influenciam o Risco de Morrer

\author{
Acta Med Port 2014:27:309-317
}

Publicado pela Acta Médica Portuguesa, a Revista Científica da Ordem dos Médicos

Av. Almirante Gago Coutinho, 151

1749-084 Lisboa, Portugal.

Tel: +351218428 215

E-mail: submissao@actamedicaportuguesa.com

www.actamedicaportuguesa.com

ISSN:0870-399X | e-ISSN: 1646-0758

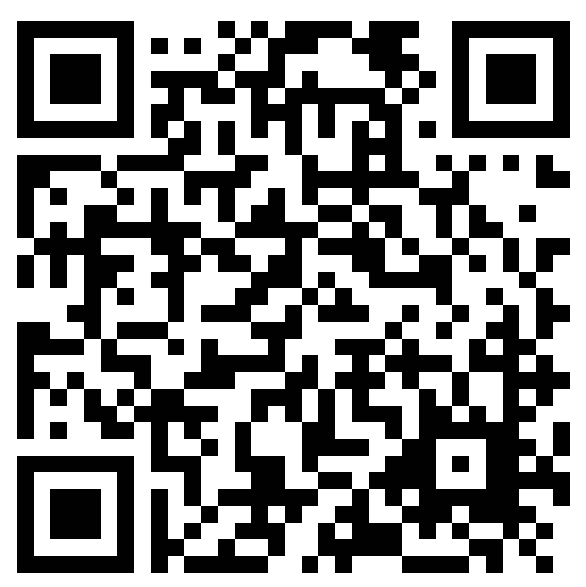

